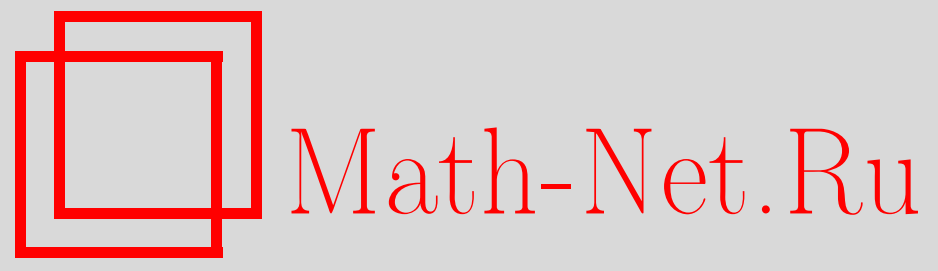

И. А. Семаев, K вопросу о сведении вычисления дискретных логарифмов на эллиптической кривой к вычислению дискретных логарифмов в конечном поле, Дискрет. матем., 1999, том 11, выпуск 3, 24-28

DOI: https://doi.org/10.4213/dm386

Использование Общероссийского математического портала Math-Net.Ru подразумевает, что вы прочитали и согласны с пользовательским соглашением http: //www . mathnet.ru/rus/agreement

Параметры загрузки:

IP : 52.23 .180 .231

26 апреля 2023 г., 12:19:22

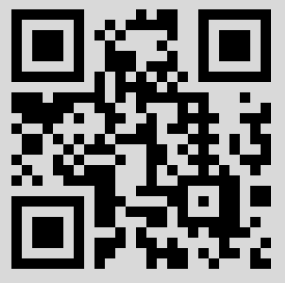




\title{
К вопросу о сведении вычисления дискретных логарифмов на эллиптической кривой к вычислению дискретных логарифмов в конечном поле
}

(c) 1999 г. И. А. Семаев

\begin{abstract}
Предлагается алгоритм вычисления вспомогательной точки, который используется в методе сведения задачи вычисления дискретных логарифмов на эллиптической кривой к вычислению логарифмов в конечном поле, предложенном ранее Менезесом, Ванстоуном, Окамото и нөзависимо автором. Алгоритм работает в более общем случае, чем соответствующий алгоритм из работы трех авторов, разработанный для случая супервырожденных кривых. Оценивается степень возникающего здесь расширения конечного поля, над которым задана эллиптическая кривая.
\end{abstract}

Пусть $E$ - заданная в форме Вейерштрасса эллиптическая кривая

$$
y^{2}+a_{1} x y+a_{3} y=x^{3}+a_{2} x^{2}+a_{4} x+a_{6}
$$

над конечным полем $F_{q}$ порядка $q$. Точки $E$ образуют коммутативную группу, которая записывается аддитивно. Точка в бесконечности $P_{\infty}$ является нулевым элементом этой группы. Пусть $E\left(F_{q^{\prime}}\right)$ - подгруппа точек $E$, определенных над $F_{q^{\prime}} \supseteq F_{q}$. Очевидно, что $E\left(F_{q^{\prime}}\right)$ - конечная коммутативная группа. Пусть $P \in E\left(F_{q}\right)$ порождает циклическую группу порядка $m$. Задача вычисления дискретных логарифмов в $\langle P\rangle$ заключается в следующем. Для данной точки $Q \in\langle P\rangle$ найти вычет $n(\bmod m)$ такой, что $n P=Q$. Задача вычисления дискретных логарифмов в конечном поле $F_{q}$ заключается в следующем. Пусть $\alpha-$ примитивный элемент поля $F_{q}$. Для данного ненулевого $\beta$ найти вычет $n(\bmod q-1)$ такой, что $\alpha^{n}=\beta$. Эти задачи имеют большое значение для криптографии [1].

Пусть $\operatorname{lcm}(m, q)=1$ и расширение $F_{q_{1}} \supseteq F_{q}$ таково, что $E\left(F_{q_{1}}\right)$ содержит подгруппу $E[m]$, изоморфную $\mathbf{Z} / m \oplus \mathbf{Z} / m$. Известно, что такое расширение существует [5]. Таким образом, $q_{1}=q^{k}$ для некоторого $k \geqslant 1$. В [2] и независимо в статье автора [3] по существу была доказана следующая теорема.

Теорема 1. Пусть задана точка $T \in E\left(F_{q_{1}}\right)$ такая, что $\langle P, T\rangle=E[m]$. Тогда сложность вычисления дискретных логарифмов в группе $\langle P\rangle$ не больше сложности вычисления дискретных логарифмов в $F_{q_{1}}$ или сложности $O(\log m)$ операций в $F_{q_{1}}$. 
Для того чтобы воспользоваться теоремой 1 , надо определть степень $k$ расширения поля $F_{q_{1}}$ над $F_{q}$, а также некоторую точку $T$, для которой $\langle P, T\rangle=E[m]$. Для супервырожденной кривой $E$ эти вопросы легко решаются. Действительно, из данных таблицы 1 в [2] следует, что $k \leqslant 6$. Там же построен вероятностный алгоритм вычисления вспомогательной точки $T$. Среднее время работы этого алгоритма ограничено полиномом от $\log q_{1}$. Примером супервырожденных кривых является кривая

$$
y^{2}=x^{3}+a_{4} x
$$

над простым полем $F_{p}$, где $p \equiv 3(\bmod 4)$, или кривая

$$
y^{2}=x^{3}+a_{6}
$$

над простым полем $F_{p}$, где $p \equiv-1(\bmod 3)$. В обоих случаях $k=2$. В общем случае пусть $G$ - максимальная подгруппа $E\left(F_{q_{1}}\right)$, порядок элементов которой есть произведение простых делителей $m$. Таким образом, $E[m] \subseteq G$ и $G=\mathbf{Z} / m_{1} \oplus \mathbf{Z} / m_{2}$, где $m$ делит $m_{1}$ и $m_{2}$. Если $m_{1}=m_{2}$, то для вычисления вспомогательной точки $T$ легко адаптировать метод статьи [2]. Заметим, что для супервырожденной эллиптической кривой это всегда так, как это следует из таблицы 1 этой статьи. В этом случае выполняется также лемма 7 из [2], которая утверждает, что если $Q-$ случайная точка на $G$, то $T=\left(m_{1} / m\right) Q$ - случайная точка на $E[m]$. Тогда при $m \rightarrow \infty$ с вероятностью, стремящейся к единице, $\langle P, T\rangle=E[m]$. Однако при $m_{1} \neq m_{2}$ легко представить себе такую ситуацию, когда последняя вероятность стремится к нулю при $m \rightarrow \infty$ (см. по этому поводу замечания в конце статьи). Таким образом, в общем случае проблема вычисления вспомогательной точки остается открытой. В статье [4], которая распространяет основные результаты статьи [2] на якобианы произвольных кривых, проблема вычисления вспомогательных точек не обсуждается.

Мы доказываем здесь следующую теорему 2 . Пусть $l$ - минимальное натуральное число, для которого $q^{l} \equiv 1(\bmod m)$.

Теорема 2. Пусть $\operatorname{lcm}(m, q-1)=1$. Тогда $k=l$ и если известно разложение $m$ на простые множители, то имеется вероятностный алгоритм вычисления точки $T \in E\left(F_{q_{1}}\right)$, для которой $\langle P, T\rangle=E[m]$. Среднее времл работы алгоритма равно $O\left(\log ^{c} q_{1}\right)$ операций в поле $F_{q_{1}}$ для некоторой постоянной с $u m \rightarrow \infty$.

Доказательство. Докажем сначала утверждение о существовании вероятностного алгоритма. Пусть $G$ - максимальная подгруппа $E\left(F_{q_{1}}\right)$, порядки элементов которой являются произведениями простых делителей $m$. Значит, $E[m] \subseteq G$. Пусть $G_{1}$ - циклическая подгруппа $G$, которая содержит некоторую точку $Q$ такую, что $\langle P, Q\rangle=E[m]$. Заметим, что это эквивалентно равенству $e_{m}(P, Q)=\mu_{m}$ для некоторого корня $\mu_{m}$ степени $m$ из единицы и спаривания $e_{m} \mathrm{~A}$. Вейля. Определение спаривания А. Вейля, а также его свойства приведены в книге [5]. Пусть $\sigma-$ эндоморфизм Фробениуса кривой $E$ над $F_{q}$, то есть отображение точек кривой в себя, индуцированное возведением их координат в степеь $Q$. Таким образом, $R^{\sigma}=R$ тогда и только тогда, когда $R \in E\left(F_{q}\right)$. Поэтому $P^{\sigma}=P$. Рассмотрим эндоморфизм $\varphi$ группы $G$, который задается соотношением

$$
R \rightarrow R \sigma-R, \quad R \in G .
$$

Так как $P \in \operatorname{Ker} \varphi$, подгруппа $\operatorname{Ker} \varphi$ нетривиальна. Рассмотрим группу $\varphi\left(G_{1}\right)$. Ясно, что $G_{1} \cap \operatorname{Ker} \varphi=P_{\infty}$, так как в противном случае $G_{1}$ содержала бы точку $T$ из 
$E\left(F_{q}\right)$ простого порядка $r$, делящего $m$, такую, что $T \in\langle Q\rangle$. Тогда по определению спаривания А. Вейля $\mu_{r} \in F_{q}$. Это противоречит условию $\operatorname{lcm}(m, q-1)=1$. Значит, $\varphi\left(G_{1}\right) \cong G_{1}$. Покажем, что

$$
\varphi\left(G_{1}\right) \cap E\left(F_{q}\right)=P_{\infty}
$$

Действительно, в противном случае $P_{1} \in \varphi\left(G_{1}\right) \cap E\left(F_{q}\right)$, где $P_{1}$ - точка простого порядка $R$, делящего $m$. Тогда $P_{1}=R^{\sigma}-R$ для некоторой точки $R \in G_{1}$. Порядок точки $R$ равен $r$, так как из равенства $(r R)^{\sigma}=(r R)$ следует, что $r R \in G_{1} \cap E\left(F_{q}\right)=$ $P_{\infty}$. Тогда из равенства $e_{r}\left(P_{1}, R+P_{1}\right)=\mu_{r}$ следуют равенства

$$
\left(\mu_{r}\right)^{\sigma}=e_{r}\left(P_{1}, R+P_{1}\right)=\mu_{r} .
$$

Значит, $q \equiv 1(\bmod r)$. Это противоречит условию теоремы. Таким образом, справедливо равенство $\varphi\left(G_{1}\right) \cap E\left(F_{q}\right)=P_{\infty}$, то есть $\varphi\left(G_{1}\right)$ содержит некоторую точку $T$, для которой $\langle P, T\rangle=E[m]$.

Пусть $T$ - минимальное натуральное число, для которого $\varphi^{t}(G)-$ циклическая группа. Очевидно, что

$$
t \leqslant \log _{2} \mid E\left(F_{q_{1}} \mid=O\left(\log q_{1}\right),\right.
$$

так как если группа $G^{\prime}=\varphi^{s}(G)$ не является циклической для некоторого $s$, то она содержит подгруппу $E[r] \cong(\mathbf{Z} / r)^{2}$ для некоторого простого делителя $r$ числа $m$. Следовательно, $G^{\prime} \cap \operatorname{Ker} \varphi \supseteq G^{\prime} \cap\langle P\rangle \neq P_{\infty}$. Поэтому порядок $\varphi\left(G^{\prime}\right)$ по меньшей мере в $r$ раз меньше порядка $G^{\prime}$.

Обозначим через $\psi$ эндоморфизм $\varphi^{t}$. Таким образом, $\psi(G)$ - циклическая группа, содержащая некоторую точку $Q$, для которой $e_{m}(P, Q)=\mu_{m}$. Сформулируем алгоритм для получения такой точки.

1. Методом статьи [6] вычислим порядок группы $E\left(F_{q_{1}}\right)$. Пусть $\left|E\left(F_{q_{1}}\right)\right|=m_{1} n$, где $n$ - максимальный делитель $\left|E\left(F_{q_{1}}\right)\right|$, для которого $\operatorname{lcm}(m, n)=1$. Легко вычислить $n$, пользуясь разложением $m$ на простые множители.

2 . Вычислим случайную точку $R \in E\left(F_{q_{1}}\right)$, решая квадратное уравнение (1) относительно $y$ при заданном случайном $x$. Это уравнение имеет решение с вероятностью $1 / 2+o(1)$ при $q_{1} \rightarrow \infty$. Оно может быть найдено посредством вероятностного алгоритма из [7]. Тогда находим $R_{1}=n R$. Так что $R_{1}-$ случайная точка в $G$, имеющая равномерное распределение на $G$ при $q_{1} \rightarrow \infty$.

3. Вычислим $U=\psi\left(R_{1} 2\right)$. Ясно, что $U-$ случайная точка на $\psi(G)$. С вероятностью, не меньшей $\phi(m) / m$ точка $U$ порождает циклическую группу порядка $m_{2}$ такого, что $m$ делит $m_{2}$, и $m_{2}$ - делитель числа $m_{1}$. Здесь $\phi(m)-$ функция Эйлера. Легко вычислить $m_{2}$, пользуясь разложением $m$ на простые множители. Значит, $T=\left(m_{2} / m\right) U$ является точкой порядка $m$, для которой $e_{m}(P, T)=\mu_{m}$.

Очевидно, что приведенный алгоритм работает в среднем полиномиальное от $\log q_{1}$ время.

Остается доказать, что $k=l$. Это утверждение вытекает из следующей леммы.

Лемма 1. Пусть выполнены условия теоремы 2 за исключением, возможно, условия $\operatorname{lcm}(m, q-1)=1$. Тогда число $l$ делит $k$ и число $k$ делит $\mathrm{lm} / v$, где

$$
v=\operatorname{lcm}\left(m,\left(q^{l}-1\right) /(q-1)\right) .
$$

Доказательство. Значения спаривания $e_{m}$ лежат в $F_{q^{l}}$, поэтому $l$ делит $k$. Пусть $P, Q$ - точки порядка $m$ такие, что $\langle P, Q\rangle=E[m]$ и $P \in E\left(F_{q}\right)$. Пусть $e_{m}(P, Q)=\mu_{m}$. 
Тогда

$$
Q^{\sigma}=u Q+s P
$$

для некоторых вычетов $u, s(\bmod m)$. Значит,

$$
e_{m}\left(P, Q^{\sigma}\right)=e_{m}(P, Q)^{\sigma}=\left(\mu_{m}\right)^{q} .
$$

С другой стороны,

$$
e_{m}\left(P, Q^{\sigma}\right)=e_{m}(P, u Q+s P)=\left(\mu_{m}\right)^{u} .
$$

Таким образом, $q \equiv u(\bmod m)$. Применим $\sigma$ к обеим частям равенства $(2)$. Так как $P^{\sigma}=P$, используя (2) снова, получаем, что

$$
Q^{\sigma^{2}}=u^{2} Q+s(u+1) P
$$

и так далее. Справедливо равенство

$$
Q^{\sigma^{l}}=u^{l} Q+s\left(u^{l-1}+\ldots+u+1\right) P=Q+\frac{s\left(q^{l}-1\right)}{q-1} P,
$$

так как $q \equiv u(\bmod m)$ и $q^{l} \equiv 1(\bmod m)$. Поэтому

$$
Q \sigma^{l m / v}=q \text {. }
$$

Таким образом, точка $Q$ определена над $F_{q} l m / v$ и, следовательно, $k$ делит $l m / v$. Лемма доказана.

Теорема 2 также доказана, так как $\operatorname{lcm}(m, q-1)=1$ и поэтому $v=m$.

Условия теоремы 2 выполнены во многих случаях. Рассмотрим теперь случай, когда $\operatorname{lcm}(m, q-1) \neq 1$. Для простоты изложения предположим, что число $m=r$ простое. Пусть максимальная $r$-подгруппа $G$ группы $E\left(F_{q}\right)$ имеет ранг 2 . Число $r$ делит $q-1$. Таким образом, группа $G$ имеет вид

$$
G=\langle R\rangle \oplus\langle S\rangle, \quad\langle R\rangle \cong \mathbf{Z} / r^{\nu}, \quad\langle S\rangle \cong \mathbf{Z} / r^{\lambda},
$$

где $1 \leqslant \nu \leqslant \lambda$, для некоторых точек $R, S$. Достаточно рассмотреть два случая, $P=r^{\nu-1} R$ и $P=r^{\lambda-1} R$, где $\lambda>\nu$. Пусть сначала $P=r^{\nu-1} R$. Тогда для случайной точки $U \in G$ вероятность того, что $e_{r}(P, Q)=\mu_{r}$, где $Q=r^{\lambda-1} U$, не меньше $1-1 / r$. Поэтому мы получаем в этом случае вероятностный алгоритм вычисления вспомогательной точки, который работает полиномиальное от $\log q$ время.

Пусть теперь $P=r^{\lambda-1} S$ и $\nu<\lambda$. Пусть для случайной точки

$$
U=U_{1}+U_{2} \in G, \quad U_{1} \in\langle R\rangle, \quad U_{2} \in\langle S\rangle,
$$

порядок $U_{1}$, равный $r^{\tau}, \tau \leqslant \nu$, не меньше, чем порядок $U_{2}$. Тогда $Q=r^{\tau-1} U-$ точка порядка $r$ такая, что $\langle P, Q\rangle=E[r]$. K сожалению, вероятность этого события не больше $1 / r$. При $r \rightarrow \infty$ эта величина мала, то есть среднее время работы такого алгоритма велико и может быть экспоненциальным от $\log q$. Однако другой путь вычисления $Q$ неизвестен.

Остается случай, когда максимальная $r$-подгруппа группы $G$ имеет ранг 1 и $r$ делит $q-1$. Этот случай сводится к случаю, когда ранг группы равен 2. Для этого надо взять более широкое поле, над которым максимальная $r$-подгруппа не является циклической. Правда, в этом случае нельзя воспользоваться первым утверждением теоремы 2, так как ее условия не выполнены. Значит, и в этом случае эффективный флгоритм вычисления вспомогательной точки остается неизвестным. 


\section{Список литературы}

1. Miller V., Use of elliptic curves in cryptography. Lect. Notes Computer Sci. (1986) 218 417-426.

2. Menezes A., Okamoto T., Vanstone S., Reducing elliptic curve logarithms to logarithms in a finite field. IEEE Trans. Inform. Theory (1993) 39, 1639-1646.

3. Семаев И. А., О вычислении логарифмов на эллиптических кривых. Дискретная математика (1996) 8, 65--71.

4. Frey G., Ruck H. G., A remark concerning $m$-divisibility and the discrete logarithms in the divisor class group of courves. Math. Comput. (1994) 62, 865-874.

5. Silverman J. H., The Arithmetic of Elliptic Curves. Springer, Berlin, 1986.

6. Schoof R., Elliptic curves over finite fields and the computation of square roots $\bmod p$. Math. Comput. (1985) 44 483-494.

7. Rabin M., Probabilistic algorithms in finite fields. SIAM J. Comput. (1980) 9, 273-280.

Статья поступила 28.05.1998. 